UCRL-JC-130753

Preprint

\title{
Effect of Divertor Geometry on Plasma Detachment in DIII-D
}

\author{
N.S. Wolf, G.D. Porter, D.N. Hill, S.L. Allen
}

This paper was prepared for submittal to 13th International Conference on Plasma Surface Interactions

May 18-22, 1998, Bahia Hotel, San Diego, CA

May 19, 1998

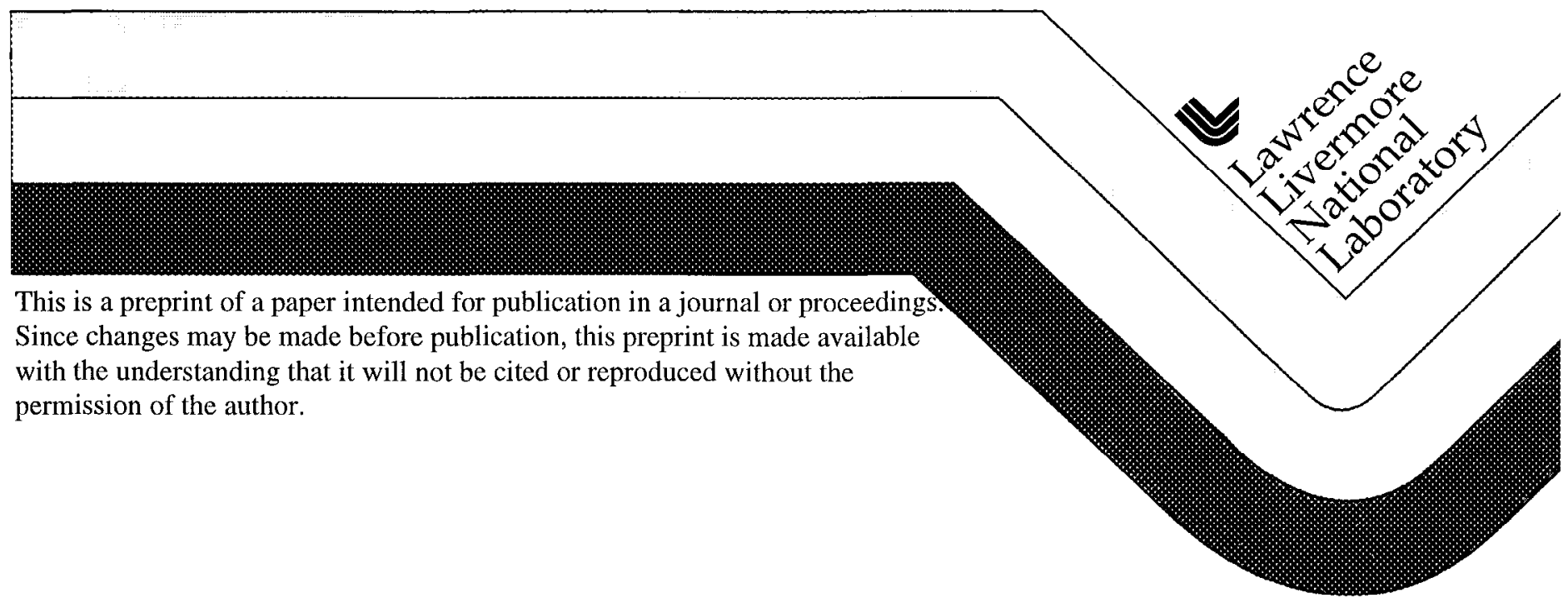




\section{DISCLAIMER}

This document was prepared as an account of work sponsored by an agency of the United States Government. Neither the United States Government nor the University of California nor any of their employees, makes any warranty, express or implied, or assumes any legal liability or responsibility for the accuracy, completeness, or usefulness of any information, apparatus, product, or process disclosed, or repiesents that its use would not infringe privately owned rights Reference herein to any specific commercial product, process, or service by trade name, trademark, manufacturer, or otherwise, does not necessarily constitule or imply its endorsement, recommendation, or favoring by the Uniled States Government or the University of California. The views and opinions of authors expressed herein do not necessatily state or reflect those of the United States Government or the University of California, and shall not be used for advertising or product endorsement purposes 


\title{
Effect of divertor geometry on plasma detachment in DIII-D
}

\author{
N. S. Wolf ${ }^{1}$, G. D. Porter ${ }^{2}$, D. N. Hill ${ }^{2}$, S. L. Allen ${ }^{2}$ \\ 'Lawrence Livermore National Laboratory and Dickinson College \\ ${ }^{2}$ Lawrence Livermore National Laboratory
}

\section{Introduction}

This paper explores the physics of the recently installed Radiative Divertor Plasma divertor (RDP) in DIII-D through the use of UEDGE simulation with experimentally derived plasma parameters. The RDP is a nearly closed bafflc and cryopumping system in the upper divertor of DIII-D. [1] One measure of the effectiveness of the RDP is the achievement of a detached plasma with a lower core density than in the open divertor (present in the lower divertor in DIII-D). Plasma detachment, observed on all diverted tokamaks, is a change in the plasma state which results in a decrease in both the ion current and heat load on the divertor plate. These reductions together with the related drop in electron temperature are important for divertor design in high power devices such as ITER, in which detached operation is assumed [2]. Both UEDGE modeling and DIII-D experiments show a reduction of $25 \%$ to $50 \%$ in the core density necessary for plasma detachment in the RDP compared to the open divertor.

\section{UEDGE modeling of detachment window in deuterium for open and RDP divertors}

The core density and SOL heating power operating range for detached plasmas in pure deuterium (no impurities) in the open, lower divertor of DIII-D was examined by Porter using UEDGE, a 2-d, hydogenic ion fluid code that includes inertial neutrals in the SOL and divertor region [3]. His technique was essentially the same as in this paper, which investigates the (nearly) closed RDP. The geometry of the problem is fixed and constant anamolous perpendicular transport coefficients are assumed. The simulations began with a best fit to experimental data from a specific discharge, and then the inputs were varied to obtain agreement with upstream density and temperature measurements, and also the divertor heat loads. For the RDP this leads to $D_{\perp}=0.3$ $\mathrm{m}^{2} / \mathrm{s}$, a wall albedo $=0.95$ and ion recycling only under the baffle of 0.95 . These input parameters were then fixed in UEDGE, and the response of the plasma to changes in the core density and SOL heating power was examined. The comparison of results for the open and closed divertors is summarized in Figure 1. 
One finds that the plasma is attached at both the inner and outer plates of the open divertor if the heating power is sufficiently high, regardless of upstream density. However, in the closed divertor we have not obtained attachment on the inside for heating powers up to $12 \mathrm{MW}$. For the open divertor, if the power is reduced, at fixed density, the inner plate plasma detaches while the outer remains attached. Detachment is defined here as a reduction of the plate temperature (at the UEDGE cell just above the strike point) below that required for efficient reionization of the recycling gas, about $5 \mathrm{eV}$. This detachment boundary does not necessarily correspond to that at which one finds dramatic reduction in the plate ion current, but we find the reduction of the plate temperature to be the first step in the detachment process.

For both the open and closed divertors, we then observe a range of heating powers at which the plasma remains detached on the inside and attached on the outside. The ionization front (defined to be at an electron temperature of $5 \mathrm{eV}$ ) moves up the inner leg towards the $\mathrm{X}$-point as the power is reduced. This movement of the ionization front expands the region with very low electron temperatures. The electron temperature below this ionization front is found to be fairly constant, between 1 and $2 \mathrm{eV}$. In DIII-D experiments, large regions with such low temperatures have been measured in the open divertor using the divertor Thomson scattering system [4], available only in the lower divertor.

The next phase of detached operation occurs for both divertors when the ionization front on the inner leg reaches the X-point. Further reduction in the heating power leads to detachment at the outer leg. The ionization front on the outer leg then moves up the separatrix, while it remains just below the X-point on the inside, as the heating power is reduced further. This is the optimum opcrating regime in which the heat load to either divertor is reduced on both the inner and outer legs. Note that for $3 \mathrm{MW}$ power for example, the core density necessary to cross this boundary is reduced from $4.5 \times 10^{19}$ in the open divertor to $2.7 \mathrm{x}^{19}$ in the RDP, a $40 \%$ decrease. This may be compared to recent DIII-D measurements that show a reduction of more than 20\%. [5]

The final phase of detached operation is reached when the ionization front on the outside lies just below the $\mathrm{X}$-point. In this state the electron temperature is between 1 and $2 \mathrm{eV}$ everywhere below the X-point and therefore neutrals, which arise from recycling at the divertor, can penetrate to the closed flux surfaces. Further reduction of the heating power leads to the core MARFE state, in which the ionization front moves above the X-point, both inside and outside. A localized high density, low temperature region forms on the closed flux surfaces just above the Xpoint on the inside. This region has sufficiently high density to radiate large amounts of power, even in the pure deuterium plasmas we have modeled.

Each of these plasma states is also seen experimentally. One always sees detached plasmas first on the inside, in fact it is rather difficult to avoid detachment there. The outer leg is usually 
detached by enhancing radiative losses with the injection of either deuterium or other gas puffing, or from sputtered carbon. Outer detachment is also seen in low power Ohmic operation. Finally, when too much gas has been injected, the core MARFE is accompanied be reduced core confinement and occasionally disruption. The similarity between these observed plasma states and those seen in the UEDGE simulation lends credence to the SOL physics models.

\section{UEDGE modeling of the detachment window in the RDP divertor with impurities}

Our UEDGE calculations explored the effect of the intrinsic carbon impurity, introduced by sputtering from the walls of DIII-D, on the RDP detachment window, assuming a fixed sputtering coefficient on all surfaces in the device. We assumed the private flux and outer walls are a source of carbon via chemical sputtering in which a low energy deuterium neutral or ion is chemically absorbed on the surface and then emerges as a volatile hydrocarbon. This molcculc is subsequently dissociated in the plasma, thus introducing carbon. The same process exists in the divertor region which is, however subject to intense deuterium ion flux near the strike points. Although bombardment with ions from an attached plasma can reach several hundred eVs, leading to higher carbon yields, the carbon sputtering for these processes is a currently an active research field. Therefore a sputtering coefficient of $Y=0.005$ was chosen to agree with upstream plasma values of DIII-D experiments.

To explore the RDP detachment boundaries with impurities we scanned the core power from $5 \mathrm{MW}$ to $10 \mathrm{MW}$ for a fixed value of core density, namely $3.0 \times 10^{19} \mathrm{~m}^{-3}$. The results are shown in Fig. 2, which presents a comparison to a RDP scan in pure deuterium. It can be seen that the intrinsic carbon impurities expand the region for a fully detached plasma and further reduce the core density necessary for detachment at a fixed power. In addition, with impurities, the RDP can reach a state of fully attached plasmas within the power range of DIII-D, which was not achieved with pure deuterium.

\section{Effect of RDP on Core Impurity Concentration}

Emplying the UEDGE code to determine the impurity transport, we started with carbon sputtered off the divertor plates, the private flux wall, and the outer wall. Two operating regimes were considered for detached inner and outer divertors; higher density operation with the lower divertor (discussed by Lasnier, at this Meeting) and lower density operation in the upper, RDP divertor. From the results of the density and power scan, we showed that it was possible to achieve detachment at half the density for a given power in the RDP as opposed to the lower divertor, therefore we examine core impurities in an upstream density of $3.0 \times 10^{19} \mathrm{~m}^{-3}$ in the RDP 
compared to $6.5 \times 10^{19} \mathrm{~m}^{-3}$ in the open divertor. The sputtering coefficients for both cases is a relatively low value of 0.002 .

The main result is that the impurity content is somewhat higher in the RDP than that found for the open divertor simulations, although the two are similar in their relationship to the detachment boundary. This undesired result can be attributed to the lower upstream density used in the RDP simulations, where the particle flux across the separatrix is less than half that of the open divertor ( $1500 \mathrm{~A}$ vs. $3700 \mathrm{~A}$, both at $3 \mathrm{MW}$ power). This leads to less parallel flow down the SOL, and hence poorer impurity entrainment. These results emphasize the importance of careful design of experiments if one wants to operate at low upstream density, as is desired for Advanced Tokamak (AT) operation in DII-D.

\section{References}

[1] S.L. Allen, et al., Proc. 24th EPS Conf., Vol. 21A, Part III, (1997) 1129

[2] G. Matthews, J. Nucl. Mat., 220-222, (1995) 104

[3] G.D. Porter, et al., Phys. Plasmas 3, (1996) 1967; T. Rognlien, et al., J. Nucl. Mat.,196198, (1992) 347

[4] S.L. Allen, et al., J. Nucl. Mat., 220-222, (1995) 336

[5] C.M. Greenfield, et al., Bull. Am. Phys. Soc., 42 (1997) 1980

*This work was performed under the auspices of the U.S. Department of Energy by Lawrence Livermore National Laboratory under contract No. W-7405-Eng-48. 


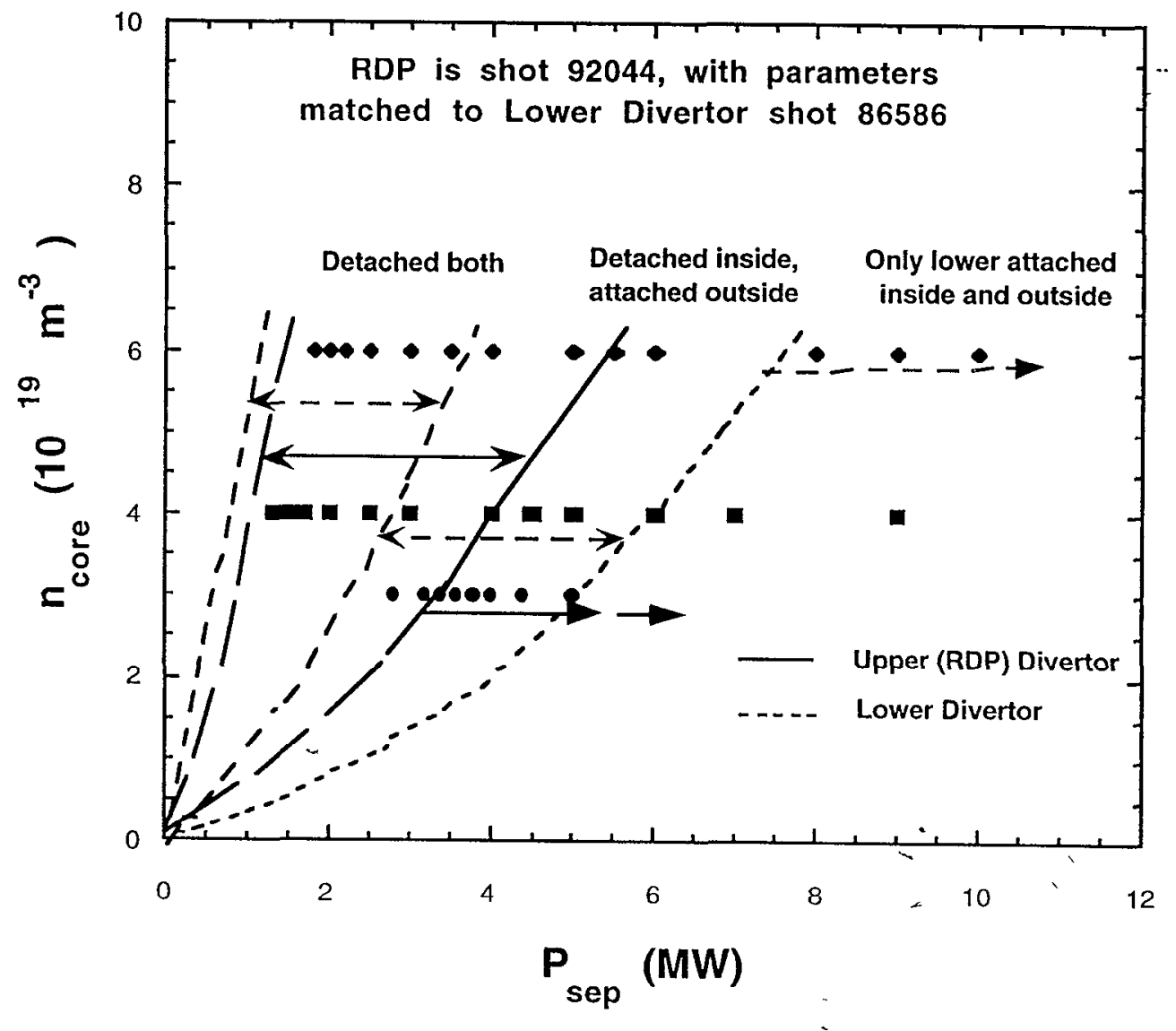

Figure 1. Detachment window for SOL plasmas in pure deuterium for open and RDP divertors in DIII-D 


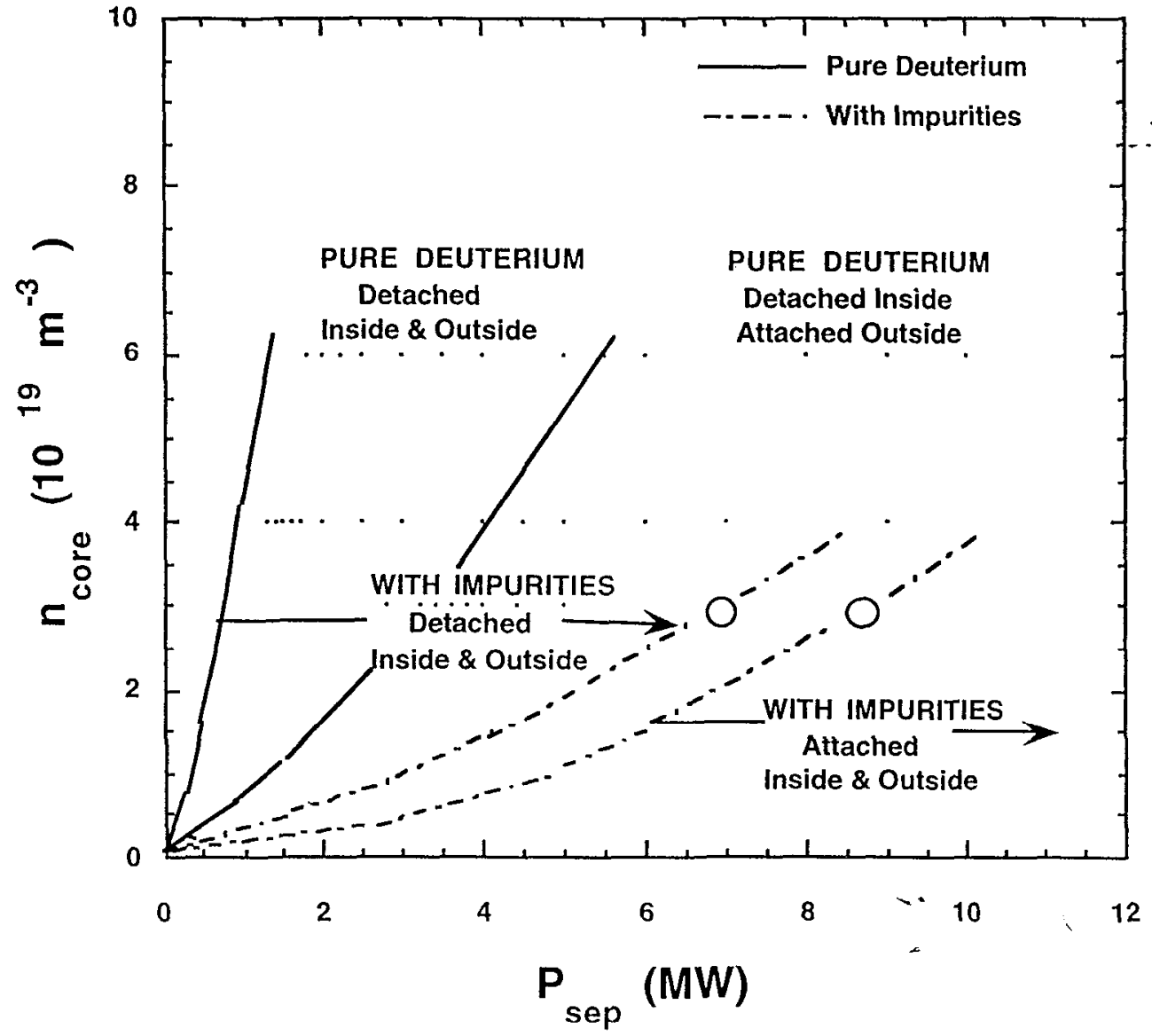

Figure 2. UEDGE simulation of the RDP detachment window for SOL plasmas with and without impurities 


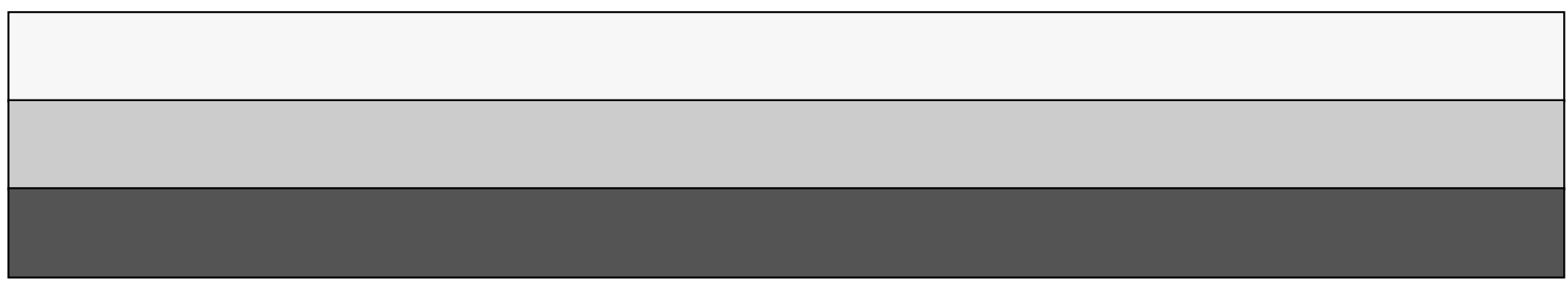

